Pacific Journal of Mathematics

A NOTE ON FRACTIONAL DERIVATIVES OF SEMIGROUPS 


\title{
A NOTE ON FRACTIONAL DERIVATIVES OF SEMIGROUPS AND COSINE FUNCTIONS
}

\author{
H. O. FATTORINI
}

It was proved by Komatsu that if $S(\cdot)$ is a strongly continuous semigroup in a Banach space $E$ then the space of all $u \in E$ such that $t \rightarrow S(t) u$ possesses a fractional derivative of order $\alpha \geq 0$ coincides with the domain of the $\alpha$ th power of (a translate of) the infinitesimal generator $A$. We prove here that a similar relationship holds for strongly continuous cosine functions, at least if $E$ belongs to a class including Hilbert spaces; in general Banach spaces only an inclusion can be assured.

1. Introduction. Let $\mathcal{Q}$ be a densely defined operator in the Banach space $E$ such that $R(\lambda ; \mathbb{Q})=(\lambda I-\mathscr{Q})^{-1}$ exists in $\lambda>0$ and satisfies

$$
\|R(\lambda ; \mathbb{Q})\| \leq c / \lambda \quad(\lambda>0) .
$$

It was shown in [1] that fractional powers $(-\mathscr{Q})^{\alpha}$ can be defined for any complex $\alpha$; we limit ourselves to real values of $\alpha$. For $0<\alpha<1(-\mathbb{Q})^{\alpha}$ is the closure of the operator

$$
\mathscr{K}_{\alpha} u=\frac{\sin \alpha \pi}{\pi} \int_{0}^{\infty} \lambda^{\alpha-1} R(\lambda ; \mathbb{Q})(-Q) u d \lambda
$$

where $D\left(\mathscr{K}_{\alpha}\right)=D(\mathbb{Q})$; for $m<\alpha<m+1(-\mathbb{Q})^{\alpha}$ is defined as the closure of $\mathscr{K}_{\alpha-m}(-\mathbb{Q})^{m}$ where $D\left(\mathscr{K}_{\alpha-m}(\mathbb{Q})^{m}\right)=D\left(\mathbb{Q}^{m+1}\right)$. The integer powers are defined as usual. See [1] for details and for the definition in the range $\alpha<0$ when $Q$ has a bounded inverse. If $A$ is an operator such that $R(\lambda ; A)$ exists in $\lambda \geq \omega_{0}$ and satisfies $\|R(\lambda ; A)\| \leq c /\left(\lambda-\omega_{0}\right)$ then $Q=A-b I$ satisfies (1.1) for $b \geq \omega_{0}$ so that the powers $(b I-A)^{\alpha}$ can be defined; it is easy to see directly from (1.2) and the first and second resolvent equation that if $b, b^{\prime} \geq \omega_{0}$ and $0<\alpha<1(b I-A)^{\alpha}-\left(b^{\prime} I-A\right)^{\alpha}$ is bounded in $D(A)$ so that

$$
D\left((b I-A)^{\alpha}\right)=D\left(\left(b^{\prime} I-A\right)^{\alpha}\right) \quad\left(b, b^{\prime} \geq \omega_{0}\right) .
$$

This equality can be extended to the whole range $\alpha \geq 0$ noting that if $m \leq \alpha<m+1$ then

$$
\begin{aligned}
D\left((b I-A)^{\alpha}\right) & =\left\{u \in D\left(A^{m}\right) ;(b I-A)^{m} u \in D\left((b I-A)^{\alpha-m}\right)\right\} \\
& =\left\{u \in D\left(A^{m}\right) ; A^{m} u \in D\left((b I-A)^{\alpha-m}\right)\right\} \\
& =\left\{u \in D\left(A^{m}\right) ;\left(b^{\prime} I-A\right)^{m} u \in D\left(\left(b^{\prime} I-A\right)^{\alpha-m}\right)\right\} .
\end{aligned}
$$


We shall denote henceforth by $F_{\alpha}$ the domain of $(b I-A)^{\alpha}$ for any $b \geq \omega_{0}$.

Let $S(\cdot)$ be a strongly continuous semigroup in $E, A$ its infinitesimal generator, $c, \omega$ constants such that

$$
\|S(t)\| \leq c e^{\omega t} \quad(t \geq 0)
$$

(so that $A$ satisfies the conditions above and $(b I-A)^{\alpha}$ can be defined at least for $b \geq \omega)$. It was proved by Komatsu [9] that $t \rightarrow S(t) u$ has a continuous fractional derivative of order $\alpha \geq 0$ if and only if $u$ belongs to $D\left((b I-A)^{\alpha}\right)$. The theory of singular perturbations of differential equations in Banach spaces ([11], [12]) leads naturally to the consideration of the same question for strongly continuous cosine functions, that is, strongly continuous operator-valued functions $C(\cdot)$ defined in $-\infty<t<\infty$ and satisfying the cosine functional equations $C(0)=I, C(s+t)+C(s-t)=$ $2 C(s) C(t)$. The infinitesimal generator $A$ of $C(\cdot)$ is defined by $A u=$ $C^{\prime \prime}(0) u$, the domain of $A$ consisting of all $u \in E$ where $t \rightarrow C(t) u$ is twice continuously differentiable. It can be shown that there exist $c, \omega \geq 0$ such that

$$
\|C(t)\| \leq c e^{\omega|t|} \quad(-\infty<t<\infty)
$$

and that $\lambda R\left(\lambda^{2} ; A\right)$ is the Laplace transform of $C(\cdot)$ for $\lambda>\omega$ so that $\|R(\lambda ; A)\| \leq c / \sqrt{\lambda}(\sqrt{\lambda}-\omega)$ there: here it follows that $Q=A-b^{2} I$ satisfies (1.1) for $b \geq \omega$, hence $\left(b^{2} I-A\right)^{\alpha}$ can be defined; again $D\left(\left(b^{2} I-A\right)^{\alpha}\right)$ is independent of $b$. (For this and other details on cosine functions see [11], [4], [5]). The identification of the subspace $E_{\alpha}$ of all $u$ where $t \rightarrow C(t) u$ has a fractional derivative of order $\alpha \geq 0$ is considerably more challenging than in the semigroup case. Except for the fact that $E_{2 n}=$ $D\left(A^{n}\right)$ (consequence of the definition of $A$ ) all that can be shown in general is that $E_{2 \alpha} \subseteq D\left(\left(b^{2} I-A\right)^{\alpha}\right)$ for $\alpha \geq 0, \alpha \neq \frac{1}{2}, \frac{3}{2}, \ldots$ (Theorem 3.1 ), the inclusion being in general strict (Remark 4.3). For $\alpha=\frac{1}{2}, \frac{3}{2}, \ldots$ neither this inclusion nor the opposite can be assured (Remarks 3.2 and 4.3). However, we can still show that $E_{2 \alpha}=D\left(\left(b^{2} I-A\right)^{\alpha}\right)$ if $E$ is a Hilbert space or more generally if $E=L^{p}$ with $1<p<\infty$ (Theorem 4.2). The proof for this case is based on a decomposition theorem for cosine functions in [5] and on Komatsu's result on groups reproduced in \$2. We point out several applications at the end of $\$ 4$ and develop one in detail.

2. Semigroups. Let $A$ be the infinitesimal generator of a strongly continuous semigroup $S(\cdot)$ satisfying (1.4). If $b>\omega$ the fractional powers 
$(b I-A)^{-\alpha}(\alpha>0)$ can be obtained directly from $S(\cdot)$ by means of the formula

$$
(b I-A)^{-\alpha} v=\frac{1}{\Gamma(\alpha)} \int_{0}^{\infty} t^{\alpha-1} e^{-b t} S(t) v d t
$$

(see [15, p. 260]); the positive powers (which coincide with those defined in $\S 1)$ are recovered through multiplication by $(b I-A)^{m}$ for sufficiently large $m$.

Fractional derivatives of $S(\cdot) u$ shall be defined by Riemann-Liouville integrals in $(t, \infty)$ rather than in $(a, t)$ to avoid dependence on the lower limit of integration (see [16, Chapter XII]): we say that $S(\cdot) u$ has a continuous derivative of order $\alpha \geq 0$ in $t \geq 0$ if and only if there exists $\beta>\omega$ and a function $f_{\beta}(\cdot)$ continuous with $s^{\alpha}\left\|f_{\beta}(s)\right\|$ integrable in $s \geq 0$ and such that

$$
e^{-\beta t} S(t) u=\frac{e^{\imath \pi \alpha}}{\Gamma(\alpha)} \int_{t}^{\infty}(s-t)^{\alpha-1} f_{\beta}(s) d s \quad(t \geq 0) .
$$

The function $f_{\beta}$ is easily seen to be unique multiplying (2.2) by $(t-r)^{-\alpha}$ and integrating in $t \geq r$; however, the present definition of fractional derivative depends on $\beta$ and on the choice of $(t, \infty)$ rather than the (equally natural interval) $(-\infty, t)$ if $S(\cdot)$ is a group (see below), thus the space of all $u \in E$ satisfying (2.2) will be called $E_{\alpha, \beta}^{+}$for the moment. That the $\beta$-dependence is actually absent follows from the next result.

THEOREM 2.1. Let $\alpha \geq 0$. Then

$$
E_{\alpha, \beta}^{+}=F_{\alpha} \quad(\beta>\omega) .
$$

Proof. The case $\alpha=0$ is obvious. Let $\alpha>0$. If $u \in F_{\alpha}$ then $u=$ $(\beta I-A)^{-\alpha} v$ so that using (2.1) and applying $S(t)$ to both sides we obtain

$$
e^{-\beta t} S(t) u=\frac{e^{i \alpha \pi}}{\Gamma(\alpha)} \int_{t}^{\infty}(s-t)^{\alpha-1} e^{-\beta s} e^{-i \alpha \pi} S(s) v d s
$$

$$
(t \geq 0, \beta>\omega)
$$

which shows that $F_{\alpha} \subseteq E_{\alpha, \beta}^{+}$. Assume now that $u \in E_{\alpha, \beta}^{+}$for some $\beta>\omega$ and let $f_{\beta}(\cdot)$ be the function in (2.2). Apply the operator $P_{\mu}=\mu R(\mu ; A)(\mu$ large enough) to both sides: we obtain the corresponding representation for $e^{-\beta t} S(t) P_{\mu} u$ with the function $P_{\mu} f_{\beta}$. However, $P_{\mu} u \in D(A) \subseteq$ $D\left((\beta I-A)^{\alpha}\right)$, hence (2.4) must hold and

$$
P_{\mu} f_{\beta}(t)=e^{-\beta t-i \alpha \pi} S(t)(\beta I-A)^{\alpha} P_{\mu} u .
$$


Write (2.5) for $t=0$ and let $\mu \rightarrow \infty$. Since $P_{\mu} f(0) \rightarrow f(0)$ and $P_{\mu} u \rightarrow u$ we use closedness of $(b I-A)^{\alpha}$ to show that $u \in D\left((b I-A)^{\alpha}\right)$. This ends the proof, and justifies using the notation $E_{\alpha}^{+}$for any $E_{\alpha, \beta}^{+}, \beta>\omega$.

The case where $S(\cdot)$ is a strongly continuous group deserves special attention. Here we require that the function $f_{\beta}$ in the definition of $E_{\alpha, \beta}$ be defined and continuous for all $t$, norm integrable at $+\infty$ and the representation (2.2) must hold in $-\infty<t<\infty$; moreover, fractional derivatives can be just as naturally defined by integrals in $(-\infty, t)$. Assume the constants $c, \omega$ are such that

$$
\|S(t)\| \leq c e^{\omega|t|} \quad(-\infty<t<\infty) .
$$

We define $E_{\alpha, \beta}^{-}$as the space of all $u \in E$ such that there exists an $E$-valued function $g_{\beta}$ continuous for all $s$, with $|s|^{\alpha}\left\|g_{\beta}(s)\right\|$ integrable at $-\infty$ and such that

$$
e^{\beta t} S(t) u=\frac{1}{\Gamma(\alpha)} \int_{-\infty}^{t}(t-s)^{\alpha-1} g_{\beta}(s) d s \quad(-\infty<t<\infty) .
$$

Make the change of variable $s \rightarrow-s$ in (2.7); formula (2.2) is obtained for $S(-t) u$ with $f_{\beta}(s)=e^{-i \pi \alpha} g_{\beta}(-s)$. Since the strongly continuous semigroup $t \rightarrow S(-t) u$ has $-A$ as infinitesimal generator, it follows that

$$
E_{\alpha, \beta}^{-}=F_{\alpha}^{-}=D\left((\beta I+A)^{\alpha}\right) \quad(\beta>\omega) .
$$

We have completed the proof of the following result.

THEOREM 2.2. Assume A generates a strongly continuous group $S(\cdot)$. Then if $\alpha \geq 0$,

$$
E_{\alpha, \beta}^{-}=F_{\alpha}^{-}, \quad E_{\alpha, \beta}^{+}=F_{\alpha} \quad(\beta>\omega) .
$$

We shall henceforth write $E_{\alpha}^{-}=E_{\alpha, \beta}^{-}(\beta>\omega)$.

REMARK 2.3. If $S(\cdot)$ is a group, (2.3) still holds if (2.2) is assumed to be valid only in $t \geq 0$ (or even in an interval $t \geq a>0$ ). This follows easily from the fact that the subspaces $F_{\alpha}$ are invariant for $S(t)$.

3. Cosine functions. Let $C(\cdot)$ be a strongly continuous cosine function with infinitesimal generator $A$ and let $c, \omega$ be constants such that (1.5) holds. Cosine functions stand in the same relation to the second order equation $u^{\prime \prime}=A u$ as semigroups to $u^{\prime}=A u$. The generator $A$ of a cosine function always generates as well a strongly continuous semigroup 
$S(\cdot)$ analytic in $\operatorname{Re} t>0$ which can be expressed from $C(\cdot)$ by means of the abstract Weierstrass formula

$$
S(t) u=\frac{1}{\sqrt{\pi t}} \int_{0}^{\infty} e^{-s^{2} / 4 t} C(s) u d s
$$

and it follows from direct estimation of (3.1) that $\|S(t)\| \leq c e^{\omega^{2} t}$ in $t \geq 0$. Accordingly, the negative fractional powers of $\left(b^{2} I-A\right), b>\omega$ can be calculated using (2.1): the same expression can be obtained using (1.2) and expressing $R(\lambda ; A)$ as a function of $C(\cdot)$ as outlined in $\S 1$. The final formula is

$$
\left(b^{2} I-A\right)^{-\alpha} u=\frac{2^{3 / 2-\alpha} b^{1 / 2-\alpha}}{\pi^{1 / 2} \Gamma(\alpha)} \int_{0}^{\infty} s^{\alpha-1 / 2} K_{\alpha-1 / 2}(b s) C(s) u d s
$$

for $\alpha>0$, where $K_{\nu}$ denotes the Macdonald function

$$
K_{\nu}(t)=\frac{\pi}{2} \frac{I_{-\nu}(t)-I_{\nu}(t)}{\sin \nu \pi}
$$

for $\nu \neq \pm \pi, \pm 2 \pi, \ldots$ and extended by continuity to all values of $\nu$ ([14, p. 78]). We note incidentally that (3.2) is a vector valued analogue of a well-known integral formula ([7, p. 763, formula 12]). The spaces $E_{\alpha, \beta}^{+} E_{\alpha, \beta}^{-}$ relative to $C(\cdot)$ are defined exactly as in the previous section for a group, that is by the relations (2.2) and (2.7) respectively, both assumed to hold in $-\infty<t<\infty$.

TheOREM 3.1. Let $\alpha \geq 0, \alpha \neq n+\frac{1}{2}, n=0,1, \ldots$ Then

$$
E_{2 \alpha, \beta}^{-} \subseteq F_{\alpha}, \quad E_{2 \alpha, \beta}^{+} \subseteq F_{\alpha} \quad(\beta>\omega) .
$$

Proof. That (3.4) holds for $\alpha=1,2,3, \ldots$ is a direct consequence of the definition of $A$. To handle the general case we note that if $\alpha>0$, $\alpha \neq n+\frac{1}{2}$, it follows from (3.3) and the power series for $I_{\nu}, I_{-\nu}$ that

$$
\begin{aligned}
t^{\alpha-1 / 2} K_{\alpha-1 / 2}(t)= & -\left(\pi / 2^{\alpha+1 / 2} \Gamma\left(\alpha+\frac{1}{2}\right) \sin \left(\alpha-\frac{1}{2}\right) \pi\right) t^{2 \alpha-1} \\
& +\rho_{\alpha}(t)
\end{aligned}
$$

where $\rho_{\alpha}(t)=t^{2 \alpha+1} \xi_{\alpha}(t)+\eta_{\alpha}(t), \xi_{\alpha}$ and $\eta_{\alpha}$ entire. Also, the asymptotic estimations of the $K_{\nu}([\mathbf{1 4}$, p. 202]) imply that

$$
K_{\nu}(t)=O\left(t^{-1 / 2} e^{-t}\right) \quad \text { as } t \rightarrow \infty
$$


with similar relations for derivatives of any order. It follows that

$$
\begin{aligned}
\sigma_{\alpha, b}(s)= & \left(2^{3 / 2-\alpha} b^{1 / 2-\alpha} / \pi^{1 / 2} \Gamma(\alpha)\right) s^{\alpha-1 / 2} K_{\alpha-1 / 2}(b s) \\
& +\left(1 / \Gamma(2 \alpha) \sin \left(\alpha-\frac{1}{2}\right) \pi\right) s^{2 \alpha-1} e^{-b s}
\end{aligned}
$$

(we have used here formula 1 in [7, p. 952]) satisfies $\sigma_{\alpha, b}(s)=O\left(s^{2 \alpha-1} e^{-b s}\right)$ as $s \rightarrow \infty$ with similar estimates for derivatives of any order; on the other hand, $\sigma_{\alpha, b}$ is continuously differentiable in $t \geq 0$, infinitely differentiable in $t>0$ and $\sigma_{a, b}^{\prime \prime}$ is integrable in $t>0$. This is easily seen to imply that $B_{\alpha, b} E \subseteq D(A)$ and that $A B_{\alpha, b}$ is bounded, where

$$
B_{\alpha, b} u=\int_{0}^{\infty} \sigma_{\alpha, b}(s) C(s) u d s
$$

it suffices to integrate by parts twice keeping in mind that $C^{\prime \prime}(t) u=$ $A C(t) u$ and use closedness of $A$. We shall denote by $J_{\alpha, b}$ the operator

$$
J_{\alpha, b} u=-\frac{1}{\Gamma(2 \alpha) \sin \left(\alpha-\frac{1}{2}\right) \pi} \int_{0}^{\infty} s^{2 \alpha-1} e^{-b s} C(s) u d s
$$

where, as before, $b>\omega$ : we have

$$
\left(b^{2} I-A\right)^{-\alpha}=J_{\alpha, b}+B_{\alpha, b} .
$$

Assume now that $0<\alpha<\frac{1}{2}$ and let $u \in E_{2 \alpha, \beta}^{+}$so that there exists an $E$-valued function $f_{\beta}(\cdot)$ continuous, with $s^{2 \alpha}\left\|f_{\beta}(s)\right\|$ integrable at infinity and such that

$$
e^{-\beta t} C(t) u=\frac{e^{2 i \alpha \pi}}{\Gamma(2 \alpha)} \int_{t}^{\infty}(s-t)^{2 \alpha-1} f_{\beta}(s) d s .
$$

Apply $P_{\mu}=\mu R(\mu ; A)$ to both sides. Since $P_{\mu} u \in D(A), t \rightarrow e^{-\beta t} C(t) P_{\mu} u$ is (twice) continuously differentiable and it is easy to show that the integral equation obtained from (3.9) can be explicitly solved by means of the formula

$$
\begin{aligned}
e^{-i \pi(1-2 \alpha)} \Gamma(1-2 \alpha) P_{\mu} f_{\beta}(t)=\int_{t}^{\infty}(s-t)^{-2 \alpha}\left(e^{-\beta s} C(s) P_{\mu} u\right)^{\prime} d s \\
=-(1-2 \alpha) \int_{t}^{\infty}(s-t)^{1-2 \alpha}\left(e^{-\beta s} C(s) P_{\mu} u\right)^{\prime \prime} d s \\
=-(1-2 \alpha) A \int_{t}^{\infty}(s-t)^{1-2 \alpha} e^{-\beta s} C(s) P_{\mu} u d s \\
\quad-2 \beta(1-2 \alpha) \int_{t}^{\infty}\left((s-t)^{1-2 \alpha} e^{-\beta s}\right)^{\prime} C(s) P_{\mu} u d s \\
-\beta^{2}(1-2 \alpha) \int_{t}^{\infty}(s-t)^{1-2 \alpha} e^{-\beta s} C(s) P_{\mu} u d s
\end{aligned}
$$


We take advantage of this equality for $t=0$. Making use of (3.8) we deduce the existence of a constant $k$ such that

$$
k P_{\mu} f(0)=A\left(\left(\beta^{2} I-A\right)^{1-\alpha}-B_{\alpha, \beta}\right) P_{\mu} u+Q_{\alpha, \beta} P_{\mu} u
$$

where $Q_{\alpha, \beta}$ is a bounded operator. Letting $\mu \rightarrow \infty$ and keeping in mind that $A$ is closed the first inclusion (3.5) follows. The second is obtained in exactly the same way using the fact that $C(t)=C(-t)$.

Consider next the case $\frac{1}{2}<\alpha<1$. Here we apply $P_{\mu}$ to both sides of (3.9), differentiate both sides with respect to $t$ and express $f_{\beta}$ as in the first step of (3.10): the result is

$$
e^{-\imath \pi(2 \alpha-1)} \Gamma(2 \alpha-1) P_{\mu} f(t)=\int_{t}^{\infty}(s-t)^{1-2 \alpha}\left(e^{-\beta s} C(s) P_{\mu} u\right)^{\prime \prime} d s
$$

so that the argument ends just as in the case $0<\alpha<\frac{1}{2}$. The range $\alpha>1$ is handled in a similar fashion.

That the argument fails when $\alpha=\frac{1}{2}, \frac{3}{2}, \ldots$ can be traced to the fact that the Macdonald function $K_{\nu}$ disgorges a logarithmic term for $\nu$ integer: in fact, if $\alpha=n+\frac{1}{2}$ we have

$$
t^{\alpha-1 / 2} K_{\alpha-1 / 2}(t)=t^{n} K_{n}(t)=c_{n} t^{2 n} \log t+r_{n}(t)
$$

where $r_{n}$ is entire. We show below that the inclusion (3.4) may fail to hold for these exceptional values of $\alpha$.

REMARK 3.2. Let $E=C_{0}(-\infty, \infty)$ be the space of all continuous functions in $-\infty<x<\infty$ such that $u(x) \rightarrow 0$ as $|x| \rightarrow \infty, C(t) u(x)$ $=\frac{1}{2}\{u(x+t)+u(x-t)\}$. It is not difficult to show that $A u=u^{\prime \prime}$ with maximal domain and that $(-A)^{1 / 2}$ is the singular integral operator

$$
(-A)^{1 / 2} u(x)=\lim _{\varepsilon \rightarrow 0} \frac{1}{2 \pi} \int_{\varepsilon \leq|s| \leq \varepsilon^{-1}} \frac{u^{\prime}(x+s)-u^{\prime}(x-s)}{s} d s
$$

Obviously $E_{1, \beta}^{+}, E_{1, \beta}^{-}(\beta>0)$ contain all continuously differentiable functions in $E$ but not all of these are in $F_{1 / 2}$. This shows the failure of (3.4) for $\alpha=\frac{1}{2}$. The argument can be easily extended to $\alpha=n+\frac{1}{2}, n=1,2, \ldots$.

That the inclusions opposite to (3.4) may not hold for any $\alpha$ will be shown in the next section (Remark 4.3).

4. Cosine functions (continuation). Let $C(\cdot), A$ be as in the previous section. It was shown in [4] that for any complex number $a, A-a^{2} I$ generates a strongly continuous cosine function $C_{a}$ with

$$
\left\|C_{a}(t)\right\| \leq c e^{(\omega+|a|) t} \quad(-\infty<t<\infty) .
$$


The cosine function $C_{a}(\cdot)$ can be obtained from $C(\cdot)$ by means of the perturbation series

$$
C_{a}(t) u=C(t) u+\sum_{n=1}^{\infty}\left(-a^{2}\right)^{n} C_{n}(t) u
$$

where $C_{n}$ is given by

$$
C_{n}(t) u=\int C\left(s_{n}\right) C\left(s_{n-1}\right) \cdots C\left(s_{1}\right) C\left(t_{0}\right) u d s d t \quad(u \in E)
$$

with $d s=d s_{1} \cdots d s_{n}, d t=d t_{0} \cdots d t_{n-1}$ and the $2 n$-dimensional integral is taken in the region $0 \leq s_{1} \leq t_{1}-t_{0}, \ldots, 0 \leq s_{n} \leq t-t_{n-1}, 0 \leq t_{0} \leq t_{1}$ $\leq \cdots \leq t_{n-1} \leq t$. Using the cosine functional equation we easily show that

$$
\left\|C_{n}(t)\right\| \leq \frac{c e^{\omega / t}|t|^{2 n}}{(2 n) !} \quad(n \geq 1)
$$

(see the details in [4, p. 94]). It is easy to see that if $n \geq 2$ each $C_{n} u$ is twice continuously differentiable for arbitrary $u \in E$; in fact, we have

$$
C_{n}^{\prime \prime}(t) u=\int C\left(t-t_{n-1}\right) C\left(t_{n-1}-t_{n-2}\right) C\left(s_{n-2}\right)
$$

$\cdots C\left(s_{1}\right) C\left(t_{0}\right) u d s^{\prime} d t$

where now $d s^{\prime}=d s_{1} \cdots d s_{n-2}, d t=d t_{0} \cdots d t_{n-1}$ and the $(2 n-2)$ dimensional integral is taken in the region $0 \leq s_{1} \leq t_{1}-t_{0}, \ldots, 0 \leq s_{n-2}$ $\leq t_{n-2}-t_{n-3}, 0 \leq t_{0} \leq t_{1} \leq \cdots \leq t_{n-1} \leq t$. We obtain from (4.5) an estimate of the type of (4.4) for the $C_{n}^{\prime \prime}$; precisely

$$
\left\|C_{n}^{\prime \prime}(t)\right\| \leq \frac{c e^{\omega|t|}|t|^{2 n-2}}{(2 n-2) !} \quad(n \geq 2)
$$

Finally, it follows also from the cosine functional equation that $C_{1} u$ is continuously differentiable with

$$
\begin{aligned}
C_{1}^{\prime}(t) u & =\frac{t}{2} C(t) u+\frac{1}{2} \int_{0}^{t} C(t-2 s) u d s \\
& =\frac{t}{2} C(t) u+\frac{1}{4} \int_{-t}^{t} C(s) u d s .
\end{aligned}
$$

It follows from (4.2) and the preceding considerations that for every $u \in E, C(t) u$ and $C_{a}(t) u$ differ by a continuously differentiable function $h$ with $\|h\|,\left\|h^{\prime}\right\|=O\left(e^{(\omega+|a|+\varepsilon) \mid t}\right)$ for any $\varepsilon>0$ so that if $E_{\alpha, \beta, a}^{+}, E_{\alpha, \beta, a}^{-}$ denote the spaces defined in the previous section relative to $C_{a}(\cdot)$ we have

$$
E_{\alpha, \beta, a}^{+}=E_{\alpha, \beta}^{+}, \quad E_{\alpha, \beta, a}^{-}=E_{\alpha, \beta}^{-} \quad(\beta>\omega+|a|)
$$


at least if $0 \leq \alpha \leq 1$. Consider next the range $1 \leq \alpha \leq 2$. If $u \in E_{\alpha, \beta}^{+}$then $u \in E_{1, \beta}^{+}$so that $C(\cdot) u$ is continuously differentiable and it follows from (4.5) and (4.4) that $C(t) u$ and $C_{a}(t) u$ differ by a twice continuously differentiable function with $\|h\|,\left\|h^{\prime}\right\|,\left\|h^{\prime \prime}\right\|=O\left(e^{(\omega+|a|+\varepsilon)|t|}\right)$ at infinity, so that (4.7) holds for $1 \leq \alpha \leq 2$. Similar arguments work for the spaces $E^{-}$ and the full range of $\alpha$. We have then proved

TRANSLATION INVARIANCE LEMma 4.1. (4.7) holds for all $\alpha \geq 0$ and all a complex.

Let $(X, \Sigma, \mu)$ be a measure space $([3, \mathrm{p} .126])$. We assume in the next result that

$$
E=L^{p}(X, \Sigma, \mu)
$$

THEOREM 4.2. Let E satisfy (4.8) with $1<p<\infty$. Then

$$
E_{2 \alpha, \beta}^{+}=E_{2 \alpha, \beta}^{-}=F_{\alpha} \quad(\beta>2 \omega)
$$

for all $\alpha>0$.

Proof. In view of Lemma 4.1 we may prove Theorem 4.2 for $E_{\alpha, \beta, a}^{+}$, $E_{\alpha, \beta, a}^{-}$with $a \geq \omega$ arbitrary. It was proved in [5] that if $a \geq \omega$ and $E$ is one of the spaces allowed in Theorem 4.2 there exists a strongly continuous group $U_{a}(\cdot)$ such that $\left\|U_{a}(t)\right\| \leq c_{\omega^{\prime}} e^{\omega^{\prime}|t|}$ for any $\omega^{\prime}>\omega+|a|$ and $C_{a}$ admits the decomposition

$$
C_{a}(t)=\frac{1}{2}\left(U_{a}(t)+U_{a}(-t)\right)
$$

The group $U_{a}(\cdot)$ has infinitesimal generator $A_{a}=i\left(a^{2} I-A\right)^{1 / 2}$ and can be expressed from $C_{a}(\cdot)$ by the formula

$$
U_{a}(t) u=C_{a}(t) u+i A_{a} \int_{0}^{t} C_{a}(s) u d s
$$

(see [5] for details). On the other hand, it follows from the results in [1] that there is a $\varphi>0$ such that $R\left(\lambda ;-\left(a^{2} I-A\right)^{1 / 2}\right)$ exists in the sector $\Sigma=\{\lambda ;|\arg \lambda| \leq \pi / 2+\varphi ; \lambda \neq 0\}$ and satisfies $\left\|R\left(\lambda ;-\left(a^{2} I-A\right)^{1 / 2}\right)\right\|$ $\leq c /|\lambda|$ in $\Sigma$; thus the operator $A_{a}$ satisfies (1.1). Accordingly, if $b \geq 0$ the fractional powers $\left(b I-A_{a}\right)^{\alpha}$ can be defined and $D\left(\left(b I-A_{a}\right)^{\alpha}\right)$ does not depend on $b$; moreover, it can be proved that if $a>\omega$ we have $\left(-A_{a}\right)^{2 \alpha}=$ $(-i)^{2 \alpha}\left(a^{2} I-A\right)^{\alpha}$. The same considerations apply to $-A_{a}=-i\left(a^{2} I-A\right)^{1 / 2}$.

Let now $u$ be an arbitrary element of $D\left(\left(a^{2} I-A\right)^{\alpha}\right)$. Then $u \in$ $D\left(\left(-A_{a}\right)^{2 \alpha}\right)$ and we can apply formula (2.4) to both the group $t \rightarrow U_{a}(t)$ 
with infinitesimal generator $A_{a}$ and to the group $t \rightarrow U_{a}(-t)$ with infinitesimal generator $-A_{a}$; in both cases we take $\beta>\omega+|a|$. Combining the two expressions thus obtained and using (4.10) we obtain formula (3.9), with

$$
f_{\beta}(t)=\frac{1}{2} e^{-\beta t} e^{-2 \imath \alpha \pi}\left(U_{a}(t)\left(\beta I-A_{a}\right)^{2 \alpha} u+U_{a}(-t)\left(\beta I+A_{a}\right)^{2 \alpha} u\right) .
$$

It follows that $u \in E_{2 \alpha, \beta, a}^{+}=E_{2 \alpha, \beta}^{+}$so that $F_{\alpha} \subseteq E_{2 \alpha, \beta}^{+}$. A similar argument applies to the spaces $E^{-}$. Combining the results obtained with Theorem 3.1 we obtain (4.9) for $\alpha \neq n+\frac{1}{2}, n=1,2, \ldots$ Note, however, that the arguments above apply as well to these exceptional values of $\alpha$; thus we only have to show that $E_{2 n+1, \beta}^{+}, E_{2 n+1, \beta}^{-} \subseteq F_{n+1 / 2}(n=0,1, \ldots)$. This has been done in [6] for $n=0$ and the result can be easily extended to $n=1,2, \ldots$; thus we omit the details. This ends the proof of Theorem 4.2.

REMARK 4.3. Let $E$ be a general Banach space, $C$ a strongly continuous cosine function in $E$. Assume that for some $a, C_{a}$ admits a decomposition of the form (4.10) where $U_{a}$ is an arbitrary strongly continuous group with infinitesimal generator $B_{a}$ (this will happen if and only if

$$
\left.A=B_{a}^{2}+a^{2} I\right) \text {. }
$$

Using the methods in Theorem 4.2 we can show that $D\left(\left(b I-B_{a}\right)^{2 \alpha}\right) \subseteq$ $E_{2 \alpha, \beta}^{+}, E_{2 \alpha, \beta}^{-}(\alpha \geq 0)$ for $b$ and $\beta$ sufficiently large. However, $B_{a}$ need not equal the "principal value" square root $i\left(a^{2} I-A\right)^{1 / 2}$; thus $D\left(\left(b I-B_{a}\right)^{2 \alpha}\right)$ may not coincide with $F_{2 \alpha}$. One such example is the space $E$ and the cosine function $C(\cdot)$ considered in Remark 3.2; here (4.10) holds for $a=0$ with $U$ the translation semigroup $U(t) u(x)=u(x-t)$; hence $A=B^{2}, B$ defined by $B u=u^{\prime}$ with maximal domain, but $B \neq(-A)^{1 / 2}$ given by formula (3.12). In those cases where no decomposition of type (4.10) exists ([8]), no obvious replacement for $F_{\alpha}$ suggests itself.

REMARK 4.4. A sort of weak version of the inclusions exists in the general case; in fact, we always have

$$
\bigcup_{\delta>0} F_{\alpha+\delta} \subseteq E_{2 \alpha, \beta}^{-}, E_{2 \alpha, \beta}^{+}
$$

for $\beta>2 \omega$ and $\alpha \geq 0$. To see this we only have to note that if $\alpha \geq \omega$, $\eta>0, v \in E$ and $U_{a}(t)$ is the operator in (4.10) then $U_{a}(t)\left(a^{2} I-A\right)^{-\delta} v$ is a continuous function of $t$ growing no more than $e^{(\omega+a+\varepsilon) t t}$ at infinity ([4]) so that if $u \in F_{\alpha+\delta}$ the representation (3.9) holds with $f_{\beta}$ given by (4.12). 
REMARK 4.5. We note that the function $f_{\beta}$ in (3.9) obtained in Theorem 4.2 for $u \in F_{\alpha}$ and $\beta>2 \omega$ dies down at infinity faster than originally bargained for: in fact, $\left\|f_{\omega}(t)\right\|=O\left(e^{-\eta t}\right)$ as $t \rightarrow \infty$ for any $\eta<\beta-2 \omega$. The same observation applies to the function $f_{\beta}$ alluded to in Remark 4.4 and, in a substantially improved version to the function in (2.3) corresponding to the semigroup case (where $\|f(t)\|=O\left(e^{-(\beta-\omega) t}\right)$ as $t \rightarrow \infty)$. Similar considerations apply to the spaces $E^{-}$.

Applications of the results to singular perturbation problems will be treated elsewhere. There are other applications, however. One is that of estimating the degree of approximation to initial conditions of (generalized) solutions of abstract second order differential equations

$$
u^{\prime \prime}(t)=A u(t) \text {. }
$$

We assume that the operator $A$ generates a cosine function $C(\cdot)$; this is known to be a necessary and sufficient condition in order that the Cauchy problem for (4.15) be well posed (strong solutions of (4.15) exist for $u(0)=u_{0}, u^{\prime}(0)=u_{1} \in D(A)$ and depend continuously on $u_{0}, u_{1}$ uniformly on compacts of $-\infty<t<\infty$; see [4], [5] for details). For $u_{0}, u_{1}$ as above the only solution of (4.15) assuming these initial conditions is

$$
u(t)=C(t) u_{0}+\int_{0}^{t} C(s) u_{1} d s .
$$

If $u_{0}, u_{1}$ are arbitrary elements of $E$ then the (continuous) function (4.16) is declared to be a generalized solution of (4.15).

THEOREM 4.6. Let $E$ be as in Theorem 4.2, $u_{0} \in F_{\alpha}, u_{1} \in F_{\gamma}$ with $\gamma=\max \left(\alpha-\frac{1}{2}, 0\right), u(t)$ the function in (4.16). Then

(a) if $0 \leq \alpha \leq 1$,

$$
\|u(t)-u(0)\|=O\left(t^{2 \alpha}\right) \quad(t \rightarrow 0)
$$

(b) if $\frac{1}{2} \leq \alpha \leq 1, u(\cdot)$ is continuously differentiable and

$$
\left\|u^{\prime}(t)-u^{\prime}(0)\right\|=O\left(t^{2 \alpha-1}\right) \quad(t \rightarrow 0) .
$$

Proof. Let $u \in F_{\alpha}$. Then $u \in E_{2 \alpha, \beta}^{+}$for any $\beta>2 \omega$ and there exists $f_{\beta}$ making (3.9) happen. We have

$$
\begin{aligned}
e^{-\beta t} C(t) u-u= & -\frac{e^{2 i \alpha \pi}}{\Gamma(2 \alpha)} \int_{0}^{t} s^{2 \alpha-1} f_{\beta}(s) d s \\
& +\frac{e^{2 i \alpha \pi}}{\Gamma(2 \alpha)} \int_{t}^{\infty}\left((s-t)^{2 \alpha-1}-s^{2 \alpha-1}\right) f_{\beta}(s) d s
\end{aligned}
$$


The first integral is obviously $O\left(t^{2 \alpha}\right)$ as $t \rightarrow 0$. As for the second, we use the easily verifiable inequality $(s-t)^{2 \alpha-1}-s^{2 \alpha-1} \leq k t^{2 \alpha}(s-t)^{-1}$ valid for $0 \leq \alpha \leq \frac{1}{2}$ obtaining a bound of the same form. Accordingly

$$
\|C(t) u-u\|=O\left(t^{2 \alpha}\right) \quad(t \rightarrow 0) .
$$

In the case $\frac{1}{2} \leq \alpha<1$ we differentiate both sides of (3.9) and apply the preceding argument to $\left(e^{-\beta t} C(t) u\right)^{\prime}$ obtaining $\left\|\left(e^{-\beta t} C(t) u\right)^{\prime}\right\|=O\left(t^{2 \alpha-1}\right)$ as $t \rightarrow 0$. An application of the mean value theorem then yields (4.20). The case $\alpha=1$ is obvious. The result just proved and another application of the mean value theorem imply that if $u \in F_{\alpha}, 0 \leq \alpha \leq 1$, then

$$
\left\|\int_{0}^{t} C(s) u d s\right\|=O\left(t^{2 \alpha+1}\right) \quad(t \rightarrow 0)
$$

if $u \in F_{\alpha}, 0 \leq \alpha \leq 1$. This relation and (4.20) are easily seen to imply the statement of Theorem 4.6.

In case $E$ is a general Banach space, the conclusion of Theorem 4.6 can be obtained under slightly stronger hypotheses on $u$, namely $u_{0} \in$ $F_{\alpha+\delta}, u_{1} \in F_{\gamma}$ with $\gamma=\max \left(\alpha+\delta-\frac{1}{2}, 0\right)$ for some $\delta>0$ (see Remark 4.4).

For other results on degree of approximation of initial conditions see $[2]$.

\section{REFERENCES}

[1] A. V. Balakrishnan, Fractional powers of closed operators and the semi-groups generated by them, Pacific J. Math., 10 (1960), 419-437.

[2] J. W. Dettman, Saturation theorems connected with the abstract wave equation, SIAM J. Math. Anal., 9 (1978), 54-64.

[3] N. Dunford and J. T. Schwartz, Linear Operators, part I, Wiley-Interscience, New York, 1958.

[4] H. O. Fattorini, Ordinary differential equations in linear topological spaces I, J. Differential Equations, 5 (1969), 72-105.

[5] Ordinary differential equations in linear topological spaces II, J. Differential Equations, 6 (1969), 50-70.

[6] Some remarks on second order abstract Cauchy problems, Funkcialaj Ekvacioj, 24 (1981), 331-344.

[7] I. S. Gradstein and I. M. Ridzyk, Tables of Integrals, Sums, Series and Derivatives, Goztekhizdat, Moscow, 1963.

[8] J. Kisyński, On operator-valued solutions of D'Alembert's functional equation, II, Studia Math., 42 (1972), 43-66.

[9] H. Komatsu, Fractional powers of operators, Pacific J. Math., 19 (1966), 285-346.

[10] S. G. Krein, Linear Differential Equations in Banach Spaces, Izdat. "Nauka", Moscow, 1967. English translation: Amer. Math. Soc. Trans. Math. Monog., vol. 29, Providence, 1971. 
[11] M. Sova, Cosine operator functions, Rozprawy Mat., 49 (1966), 1-47.

[12] __ Équations hyperboliques avec petit paramètre dans les espaces de Banach généraux, Colloq. Math., 21 (1970), 303-320.

[13] __ Encore sur les équations hyperboliques avec petit paramètre dans les espaces de Banach généraux, Colloq. Math., 25 (1972), 135-161.

[14] G. N. Watson, A Treatise on the Theory of Bessel Functions, Cambridge University Press, Cambridge, 1944.

[15] K. Yosida, Functional Analysis, 5th ed., Springer, Berlin, 1978.

[16] A. Zygmund, Trigonometric Series, Cambridge University Press, Cambridge, 1959.

Received May 8, 1981 and in revised form May 28, 1982. This work was supported in part by the National Science Foundation under Grant MCS79-03163.

UNIVERSITY OF CALIFORNIA

Los ANGELES, CA 90024 



\title{
PACIFIC JOURNAL OF MATHEMATICS EDITORS
}

\author{
Donald BabBitT (Managing Editor) \\ University of California \\ Los Angeles, CA 90024 \\ Hugo RossI \\ University of Utah \\ Salt Lake City, UT 84112 \\ C. C. Moore and Arthur Ogus \\ University of California \\ Berkeley, CA 94720
}

J. DugundiI

Department of Mathematics

University of Southern California

Los Angeles, CA 90089-1113

R. FinN and H. SAMELSON

Stanford University

Stanford, CA 94305

\section{ASSOCIATE EDITORS}
R. ARENS
E. F. BECKENBACH
B. H. NeUmanN
F. WOLF
K. YosHida
(1906-1982)

\section{SUPPORTING INSTITUTIONS}

\author{
UNIVERSITY OF ARIZONA \\ UNIVERSITY OF BRITISH COLUMBIA \\ CALIFORNIA INSTITUTE OF TECHNOLOGY \\ UNIVERSITY OF CALIFORNIA \\ MONTANA STATE UNIVERSITY \\ UNIVERSITY OF NEVADA, RENO \\ NEW MEXICO STATE UNIVERSITY \\ OREGON STATE UNIVERSITY
}

\author{
UNIVERSITY OF OREGON \\ UNIVERSITY OF SOUTHERN CALIFORNIA \\ STANFORD UNIVERSITY \\ UNIVERSITY OF HAWAII \\ UNIVERSITY OF TOKYO \\ UNIVERSITY OF UTAH \\ WASHINGTON STATE UNIVERSITY \\ UNIVERSITY OF WASHINGTON
}

The Supporting Institutions listed above contribute to the cost of publication of this Journal, but they are not owners or publishers and have no responsibility for its content or policies.

Mathematical papers intended for publication in the Pacific Journal of Mathematics should be in typed form or offset-reproduced (not dittoed), double spaced with large margins. Please do not use built up fractions in the text of the manuscript. However, you may use them in the displayed equations. Underline Greek letters in red, German in green, and script in blue. The first paragraph must be capable of being used separately as a synopsis of the entire paper. In particular it should contain no bibliographic references. Please propose a heading for the odd numbered pages of less than 35 characters. Manuscripts, in triplicate, may be sent to any one of the editors. Please classify according to the scheme of Math. Reviews, Index to Vol. 39. Supply name and address of author to whom proofs should be sent. All other communications should be addressed to the managing editor, or Elaine Barth, University of California, Los Angeles, California 90024.

There are page-charges associated with articles appearing in the Pacific Journal of Mathematics. These charges are expected to be paid by the author's University, Government Agency or Company. If the author or authors do not have access to such Institutional support these charges are waived. Single authors will receive 50 free reprints; joint authors will receive a total of 100 free reprints. Additional copies may be obtained at cost in multiples of 50 .

The Pacific Journal of Mathematics is issued monthly as of January 1966. Regular subscription rate: $\$ 132.00$ a year (6 Vol., 12 issues). Special rate: $\$ 66.00$ a year to individual members of supporting institutions.

Subscriptions, orders for numbers issued in the last three calendar years, and changes of address should be sent to Pacific Journal of Mathematics, P.O. Box 969, Carmel Valley, CA 93924, U.S.A. Old back numbers obtainable from Kraus Periodicals Co., Route 100, Millwood, NY 10546.

The Pacific Journal of Mathematics ISSN $0030-8730$ is published monthly by the Pacific Journal of Mathematics at P.O. Box 969, Carmel Valley, CA 93924. Application to mail at Second-class postage rates is pending at Carmel Valley, California, and additional mailing offices. Postmaster: Send address changes to Pacific Journal of Mathematics, P. O. Box 969, Carmel Valley, CA 93924.

PUBLISHED BY PACIFIC JOURNAL OF MATHEMATICS, A NON-PROFIT CORPORATION

Copyright $\odot 1983$ by Pacific Journal of Mathematics 


\section{Pacific Journal of Mathematics}

Vol. 109, No. $2 \quad$ June, 1983

Tibor Bisztriczky, On the singularities of almost-simple plane curves . . . . 257

Peter B. Borwein, On Sylvester's problem and Haar spaces .............. 275

Emilio Bujalance, Cyclic groups of automorphisms of compact nonorientable Klein surfaces without boundary ............... 279

Robert Jay Daverman and John J. Walsh, Acyclic decompositions of

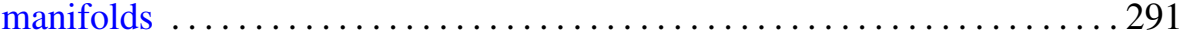

Lester Eli Dubins, Bernstein-like polynomial approximation in higher

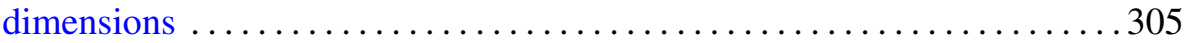

Allan L. Edelson and Jerry Dee Schuur, Nonoscillatory solutions of

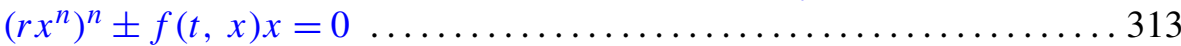

Akira Endô, On units of pure quartic number fields ................. 327

Hector O. Fattorini, A note on fractional derivatives of semigroups and

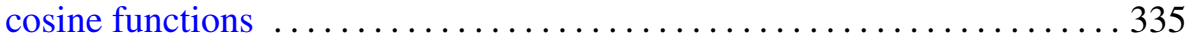

Ronald Fintushel and Peter Sie Pao, Circle actions on homotopy spheres with codimension 4 fixed point set ........................ 349

Stephen Michael Gagola, Jr., Characters vanishing on all but two

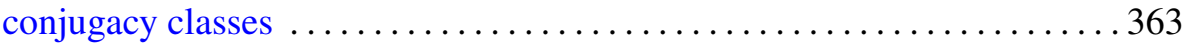

Saverio Giulini, Singular characters and their $L^{p}$ norms on classical Lie

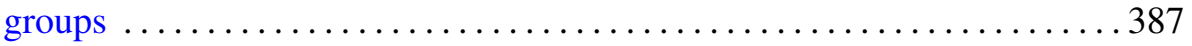

Willy Govaerts, Locally convex spaces of non-Archimedean valued continuous functions

Wu-Chung Hsiang and Bjørn Jahren, A remark on the isotopy classes of diffeomorphisms of lens spaces $\ldots . \ldots \ldots \ldots \ldots \ldots$

Hae Soo Oh, Compact connected Lie groups acting on simply connected 4-manifolds

Frank Okoh and Frank A. Zorzitto, Subsystems of the polynomial system

Knut Øyma, An interpolation theorem for $H_{E}^{\infty}$

Nikolaos S. Papageorgiou, Nonsmooth analysis on partially ordered vector spaces. II. Nonconvex case, Clarke's theory ........ 